\title{
A Novel Synthesis of Polyisoxazoline and Polyisoxazole. The Reaction of Terephthalohydroxamoyl Chloride with Bisdipolarophiles
}

\author{
Yoshio Iwakura, Keikichi Uno, Suck-Ju Hong, \\ and Tatsuhiko Hongu \\ Department of Synthetic Chemistry, Faculty of Engineering, The University \\ of Tokyo, Hongo, Bunkyo-ku, Tokyo, Japan.
}

(Received August 11, 1970)

\begin{abstract}
The same polyisoxazoline and polyisoxazole as routine 1,3-dipolar polycycloadducts were synthesized in good yields by refluxing terephthalohydroxamoyl chloride (TPHC), the precursor of terephthalonitrile di- $N$-oxide, with bisdipolarophiles in a polar or nonpolar solvent. TPHC was condensed with $m$ - and $p$-phenylenebismaleimides, hydroquinone bismethacrylate, ethylenebisacrylamide and bisphenol-A bismethacrylate resulting in the formation of polyisoxazolines. Also the reaction of TPHC with $p$-divinylbenzene yielded the corresponding isoxazoline polymer. Polyisoxazoline from hydroquinone bismethacrylate gave the highest value of reduced viscosity among the polymers obtained. Polyisoxazoline from ethylenebismethacrylamide showed a fairly good crystallinity. Polyisoxazole (VII-A) from the reaction of TPHC with 1,4-diethynylbenzene in toluene gave the highest crystallinity among the polymers obtained. Polyisoxazoline (II-A) from $m$-phenylenebismaleimide and polyisoxazole (VIIA) showed a polyelectrolytic viscosity behavior in $98-\%$ sulfuric acid.

KEY WORDS 1,3-Dipolar Polycycloadducts / Terephthalohydroxamoyl Chloride / Polyisoxazoline / Polyisoxazole / Crystallinity / 1,4-Diethynylbenzene / $p$-Divinylbenzene / Ethylene Bismethacrylate / Hydroquinone Bismethacrylate / Bisphenol-A Bismethacrylate / Ethylenebismethacrylamide / $p$ - and $m$-Phenylenebismaleimde / Polyelectrolytic Viscosity Behaviors /
\end{abstract}

Since the isolation of dinitrile $N$-oxide, ${ }^{1} 1,3$ dipolar polycycloadditions of terephthalonitrile di- $N$-oxide with diolefins ${ }^{2}$ were carried out for the synthesis of polyisoxazolines in our laboratory. But the dinitrile $N$-oxide was apt to form furoxane, thus lowering the degree of polymerization of the polycycloadducts with the exception of a few examples. Recently, Sasaki, et al., ${ }^{3}$ have reported that hydroxamoyl chloride with monofunctional dipolarophiles forms the same products as ordinary 1,3-dipolar cycloadducts. Accordingly, we have now utilized this direct reaction method to prepare polyisoxazolines and polyisoxazole.

In the present paper, we wish to describe the preparation of the same polyisoxazolines (I, II, and VI) and polyisoxazole (VII) from the reactions of TPHC with the corresponding dipolarophiles, as those via 1,3-dipolar polycyclo- additions, ${ }^{2,4}$ and the synthesis of new polyisoazolines (III, IV, V, and VIII) by the present polymerization method. Polyisoxazole (VII) obtained from the reaction of TPHC with 1,4-diethynylbenzene was found to be highly crystalline. Polyisoxazole (VIII-A) and polyisoxazoline (II-A) showed polyelectrolytic viscosity behavior in $98-\%$ sulfuric acid.

\section{RESULTS AND DISCUSSION}

The reactions of TPHC with bisdipolarophiles were carried out in toluene or anisole at their refluxing temperatures. During the initial period of the reaction, vigorous evolution of hydrogen chloride was always observed, and usually the polymer precipitated gradually in the course of the reaction. The end of the reaction was determined by testing the chlorine in the reaction 
mixture by the Beilstein method. After the reaction was completed, the precipitates were purified by reprecipitation, and their viscosities were measured in an Ubbelohde viscometer.

Sasaki, et al. ${ }^{3}$ have postulated that the reaction of monofunctional hydroxamoyl chloride with dipolarophile might proceed via the adduct having a structure favoring simultaneous dehydrochlorination to give the same product as that via nitrile oxide. In the present work, because the reaction of TPHC with bisdipolarophile could be assumed as the extended bisfunctional reaction after Sasaki, polymerizations of TPHC with bisdipolarophiles could proceed via multicentered adducts (T-A) as shown in the following scheme.

Table I summarizes the results of the reactions of TPHC with diolefins and 1,4-diethynylbenzene.

When an anisole was used as a polar reaction medium, it appeared that a more comparatively homogeneous reaction took place than in the case of toluene; a generally higher degree of polymerization was attained, but polymer yields were lower than in the case of toluene. This might be due to the fact that lower molecular weight portions were dissolved in anisole.

The polymers VIII-A and the greater part of the polymer VII-B were soluble only in $98-\%$ sulfuric acid among the solvents tested, giving a strong green fluorescence. About $30 \%$ of VII-B was insoluble even in $98-\%$ sulfuric acid, presumably owing to the cross linking reaction of the polymer at elevated temperatures in anisole.

The polymers obtained from the reactions were filtered, washed thoroughly with methanol and dried. The dried polymers were then confirmed to be sufficiently pure as those reprecipitated. The polymers were then tested for their XRD patterns. Figure 1 shows the X-ray diffraction diagrams for III-A, III-B, VI-A, and VI-B. While VI-A and $B$, the reaction products of TPHC with ethylene bismethacrylate, are amorphous, the III-A and B obtained from hydroquinone bismethacrylate show fairly good crystallinity.

$\mathrm{V}-\mathrm{A}$ and $\mathrm{B}$ having amide bounding, are highly crystalline as shown in Figure 2.

Synthesis of pelyisoxazole of higher molecular

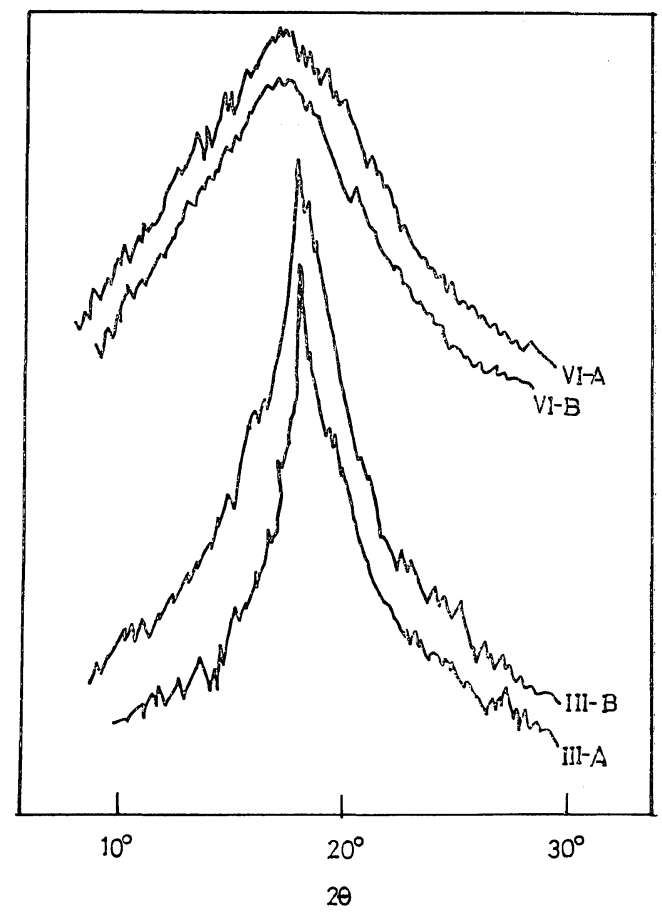

Figure 1. The X-ray diffraction diagrams of IIIA, III-B, VI-A, and VI-B.

weight by Overberger ${ }^{4}$ was very interesting, but we could not find references to its crystallinity. We have obtained highly crystalline polyisoxazole from the reaction of TPHC with 1,4-diethynylbenzene in toluene or anisole as shown in Figure 3. The 1,3-dipolar polycycloadduct of terephthalonitrile $\mathrm{di}-\mathrm{N}$-oxide with 1 ,4-diethynylbenzene in toluene (VII-C, $\eta_{\mathrm{sp}} / \mathrm{C}=0.67$ in $0.5 \mathrm{~g} / \mathrm{d} l$ of $98-\%$ sulfuric acid at $30^{\circ} \mathrm{C}$ ) was found to be highly crystalline, indicating the same position of crystalline peaks as VIII-A and B. But we could also find significant differences of the crystallinities among the polymers, VII-A, B, and C; the degree of crystallinity decreased in the order of VII-A $>$ VII-C $>$ VII-B.

VII-B containing 30-\% insoluble portions in 98-\% sulfuric acid showed the lowest crystallinity. In the case of the VII-C, the precipitation of the polymer occurred within a few minutes in the course of the reaction. Such rapid precipitation of the polymer increases the possibility of the random alignment of the polymer chains, thus lowering the crystallinity 
Y. Iwakura, K. Uno, S. Hong, and T. Hongu

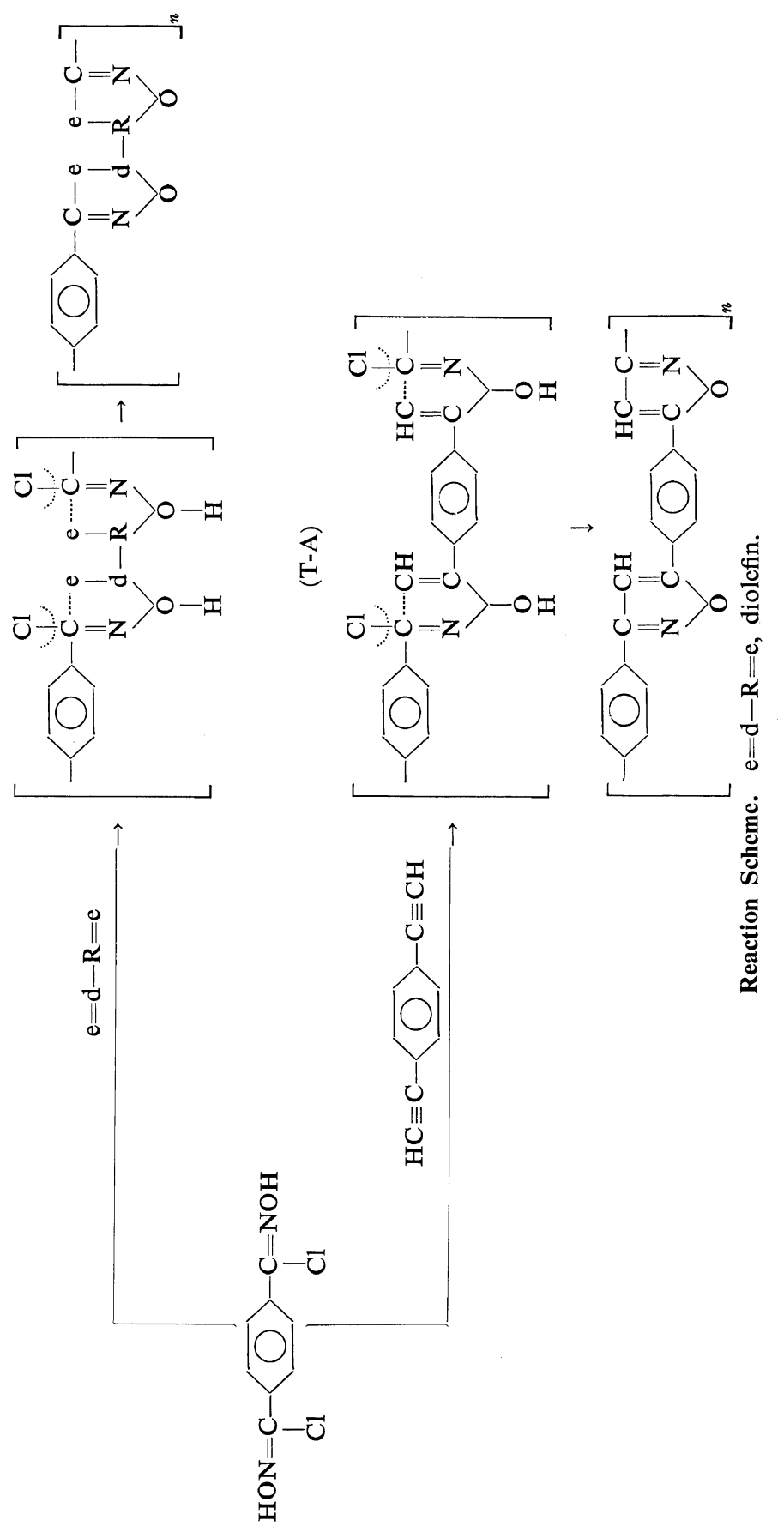


A Novel Synthesis of Polyisoxazoline and Polyisoxazole: The Reaction of TPHC with Bisdipolarophiles

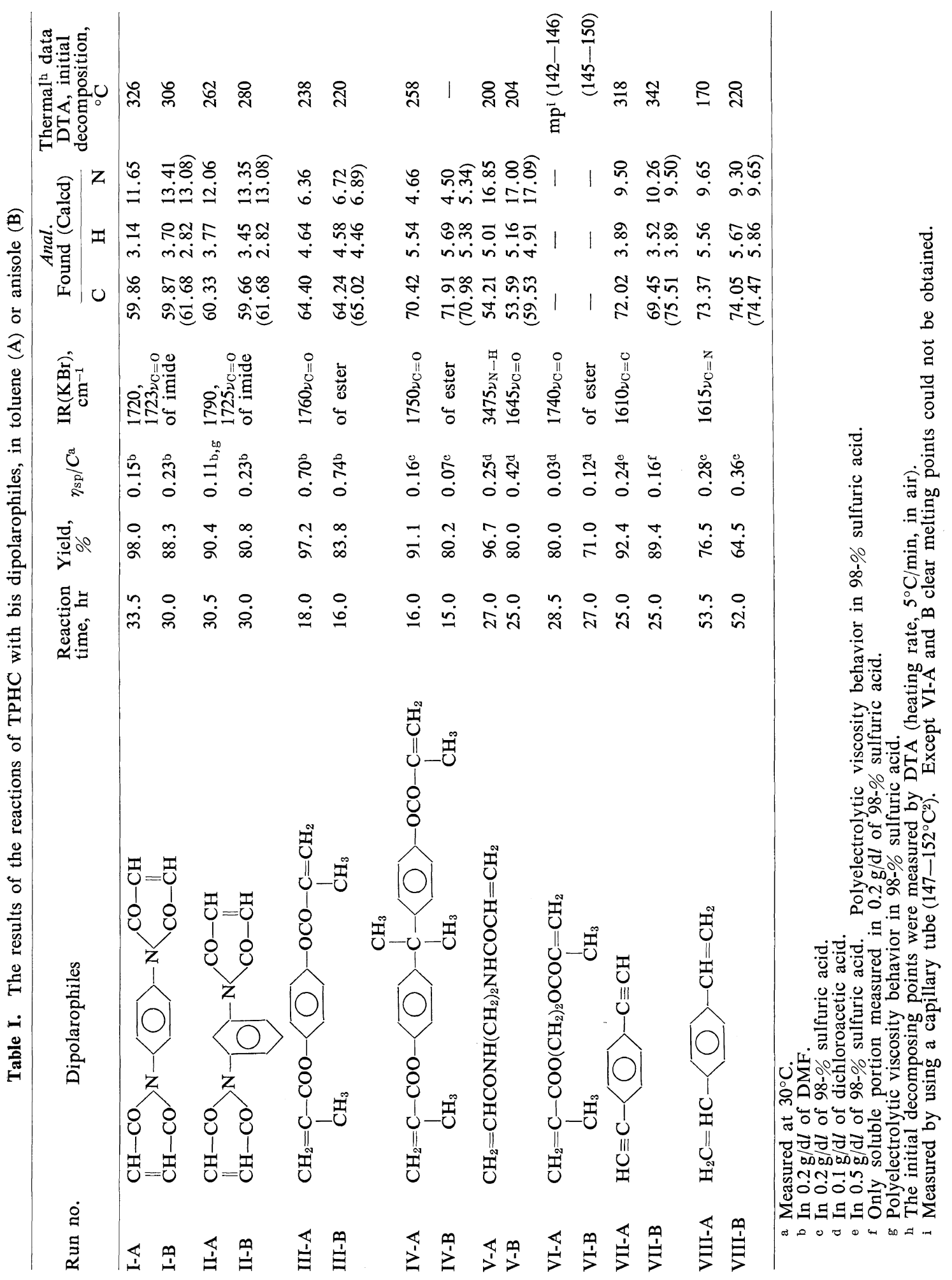




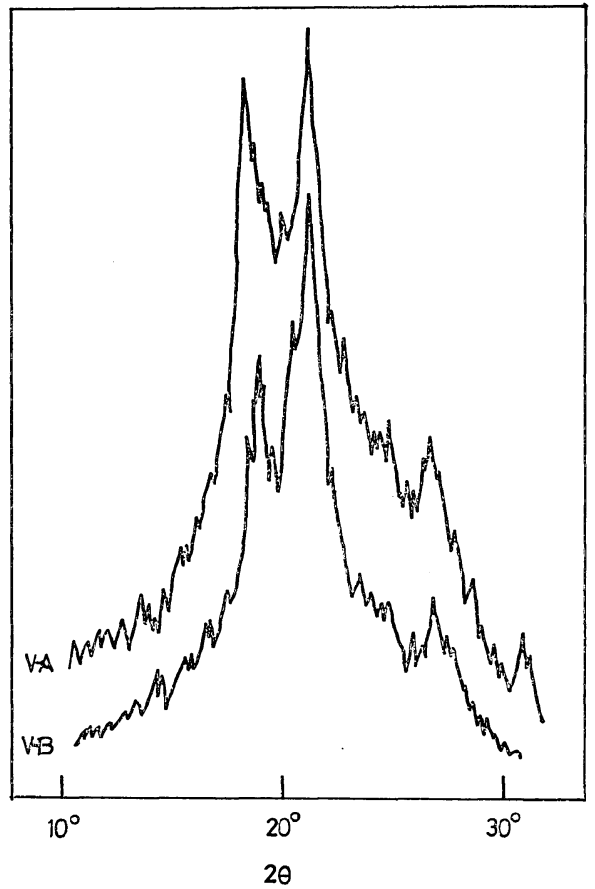

Figure 2. The X-ray diffraction diagrams of V-A, and $\mathbf{B}$.

of the polymer. However, VII-A was precipitated more slowly without the cross-linking reaction of the polymer in the course of the reaction than in the case of VII-C. Such comparatively slow precipitation of the polymer would contribute greatly to the growth of the regular chain alignment of the polymer. The crystallinity of VII-A was therefore; highly facilitated. It may be reasonable to assume that VII-C has a lower crystallinity, owing to its relative precipitation rate in the course of the reaction.

In the present work, it was found that TPHC was more convenient than terephthalonitrile di$N$-oxide obtained from the dehydrochlorination of TPHC, for the purpose of increasing the crystallinity of polyisoxazole. Toluene was a convenient reaction medium for promoting the crystallinity of polyisoxazole, because the crosslinking reaction of the polymer might occur in anisole and lower the crystallinity of the produced polymer such as VII-B. VII-A dissolved in 98-\% sulfuric acid, gave a precipitate when poured into water. The recovered polyisoxa-

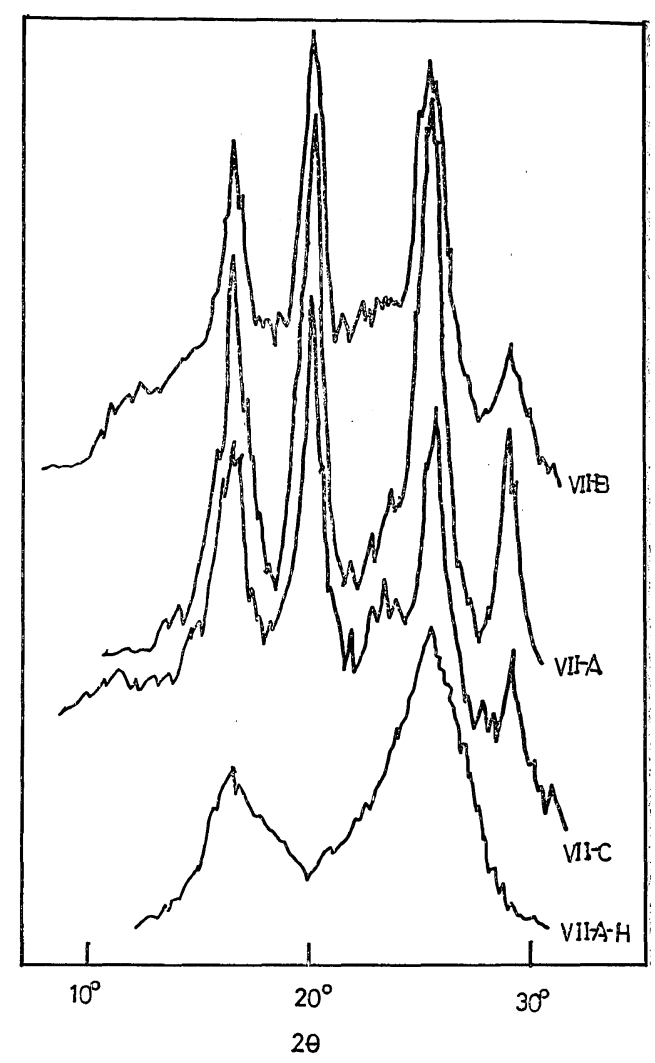

Figure 3. The X-ray diffraction diagrams of polyisoxazole.

zole (VII-A-H) gave a much different XRD pattern from that of the original polymer as shown in Figure 3. The polymer samples. were used for the measurements of the X-ray diffraction diagrams without any thermal pretreatment. As shown in Figure 3, such a. change of XRD pattern by reprecipitation from the crystalline polymer (VII-A) could be due to the increase of random alignment of the polymer chains, which might be caused from instantaneous precipitation in the course of polymer recovery.

Since little attention has hitherto been paid to the viscosity behavior of the polymers with heterocyclic rings such as polyisoxazoline and polyisoxazole, we would like also to present our experimental results on the viscosity behavior of II-A and VII-A in $98-\%$ sulfuric acid. Viscosities were measured in an Ubbelohde: viscometer at $30^{\circ} \mathrm{C}$. The reduced viscosity- 
A Novel Synthesis of Polyisoxazoline and Polyisoxazole: The Reaction of TPHC with Bisdipolarophiles

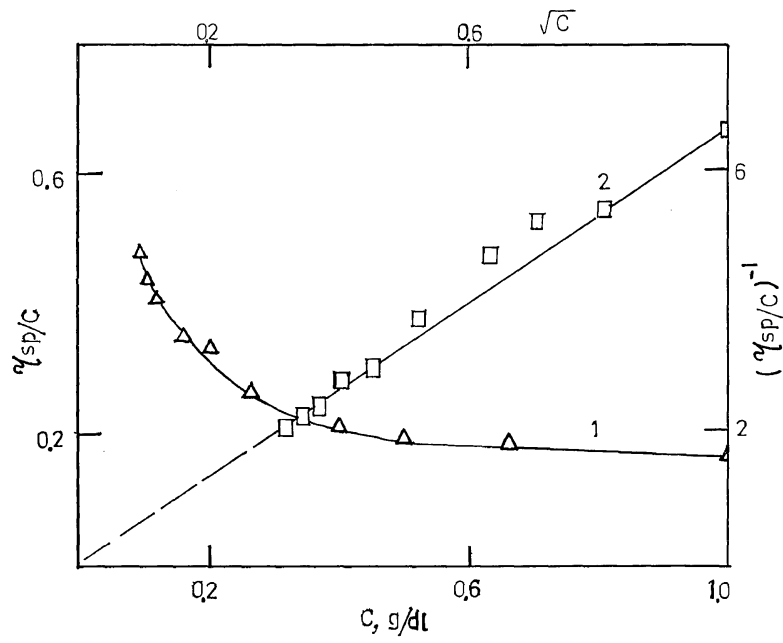

Figure 4. Reduced viscosity-concentration curves for II-A: 1, in $98-\%$ sulfuric acid: 2, plotted according to the Fuoss equation.

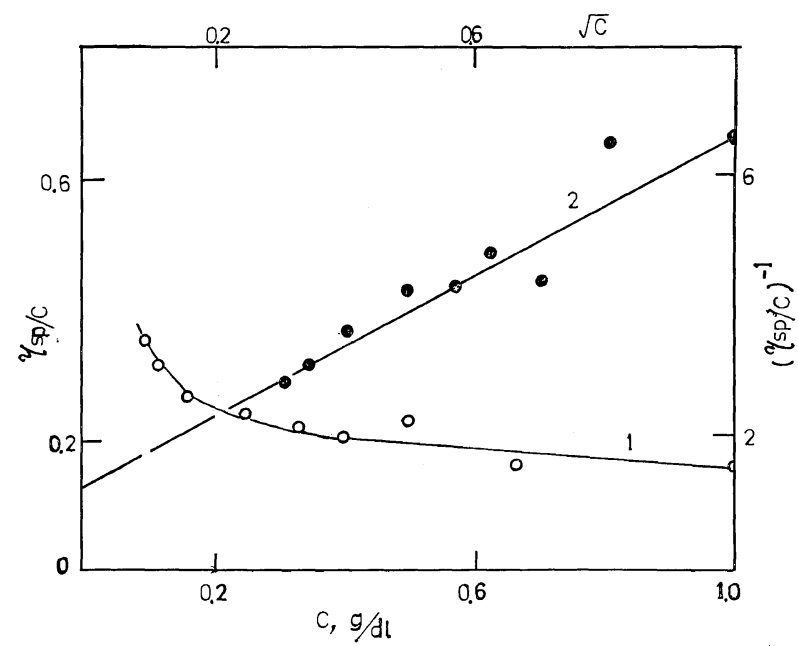

Figure 5. Reduced viscosity-concentration curves for VII-A: 1, in $98-\%$ sulfuric acid: 2, plotted according to the Fuoss equation.

concentration curves for II-A and VII-A in 98$\%$ sulfuric acid are shown in Figures 4 and 5, respectively.

The observed viscosities of II-A and VII-A increased with dilution as shown in Figures 4 and 5. The viscosity data observed in $98-\%$ sulfuric acid were also plotted according to the Fuoss empirical equation as shown in Figures 4 and 5.

$$
\eta_{\mathrm{sp}} / C=A /\left(1+B C^{1 / 2}\right)
$$

Polyisoxazoline (II-A) and polyisoxazole (VII-
Table II. The physical constants of the Fuoss empirical equation for II-A, and VII-A

\begin{tabular}{lcc}
\hline Run no. & $A^{\mathrm{a}}$ & $B^{\mathrm{b}}$ \\
\hline II-A & 10.0 & 6.25 \\
VII-A & 0.74 & 0.36 \\
\hline
\end{tabular}

a The limiting viscosity numbers extrapolated to zero concentration, the analog of ordinary neutral polymer.

b Electrostatic forces between polyions, and counter ions, obtained from the value of $A \times$ slope $(B / A)$ in Figure 4 (curve 2), and 5 (curve 2). 
A) clearly showed polyelectrolytic viscosity behavior in 98-\% sulfuric acid. The physical constants for II-A and VII-A calculated according to the Fuoss equation are summarized in Table II.

\section{EXPERIMETAL}

\section{Materials}

Solvents used were purified by the usual method.

Terephthalohydroxamoyl chloride, ${ }^{1} \mathrm{mp} 185^{\circ} \mathrm{C}$. $p$-Phenylenedimaleimides (A) and $m$-Phenylenedimaleimides $(B)^{6}$ were prepared by the conventional method. A did not melt at the temperature above $380^{\circ} \mathrm{C}$ (1it. ${ }^{6} \mathrm{mp} 346-350^{\circ} \mathrm{C}$ ), and was identified by the IR spectrum. $\mathrm{mp}$ for B $198-199^{\circ} \mathrm{C}\left(\right.$ lit. $\left.^{6} \mathrm{mp} 202^{\circ} \mathrm{C}\right)$.

1,4-Diethynylbenzene ${ }^{7}$ was prepared by bromination of $p$-divinylbenzene followed by dehydrobromination, then purified by sublimation mp $95.5-96^{\circ} \mathrm{C}$.

$p$-Divinylbenzene was purified by distillation under reduced pressure. bp $49^{\circ} \mathrm{C}(2 \mathrm{~mm})$.

\section{Polymerization}

A typical example of polymerization procedure is as follows: TPHC $(0.002 \mathrm{~mol})$ and equimolecular amount of 1,4-diethynylbenzene freshly purified were refluxed in $5 \mathrm{ml}$ of toluene until the evolution of hydrogen chloride ceased. As the reaction proceeded the polymer was precipitated slowly. After the reaction was completed the resulting reaction mixture was cooled to room temperature and filtered, washed thoroughly with hot methanol, then dried under reduced pressure at room temperature for three days to afford light brown colored powder in $92.4-\%$ yields.

\section{Measurement}

The infrared spectrum was measured on an Hitachi-EPI-S-2 type spectrophotometer.

The X-ray diffraction diagrams were obtained by the powder method with use of nickel-filtered CuK radiation, using a Rigaku Denki X-ray diffractometer.

Thermal data (DTA) were measured by use of Rigaku Denki thermogravimetric and differential thermal analyzers.

Acknowledgement. The authors are grateful to Dr. N. Nakabayashi for kindly supplying bisphenol-A bismethacrylate, hydroquinone bismethacrylate, ethylenebisacrylamide and ethylenebismethacrylate.

\section{REFERENCES}

1. Y. Iwakura, M. Akiyama, and K. Nagakubo, Bull. Chem. Soc., Japan, 37, 767 (1964).

2. Y. Iwakura, S. Shiraishi, M. Yuyama, ibid., 41, 1648 (1968).

3. T. Sasaki, and T. Yoshioka, ibid., 40, 2604 (1967).

4. C. G. Overberger, and S. Fujimoto, Polymer. Letters, 3, 735 (1965).

5. R. M. Fuoss, J Polym. Sci., 3, 603 (1948).

6. P. Kovacic, and R. W. Hein, J. Amer. Chem. Soc., 81, 1187 (1959).

7. A. S. Hay, J. Org. Chem., 25, 637 (1960). 